\title{
Total Internal Reflection Transient Absorption Microscopy: An On-line Detection Method for Microfluidics
}

\section{SUPPLEMENTARY INFORMATION}

Brandon A. Colon, Muhammad Redwan Hassan, Amirus Saleheen, Christopher A. Baker, and

Tessa R. Calhoun*

Department of Chemistry, University of Tennessee, Knoxville, Tennessee, 37996, United States

\section{Corresponding Authors}

*Tessa R. Calhoun, Email: trcalhoun@utk.edu
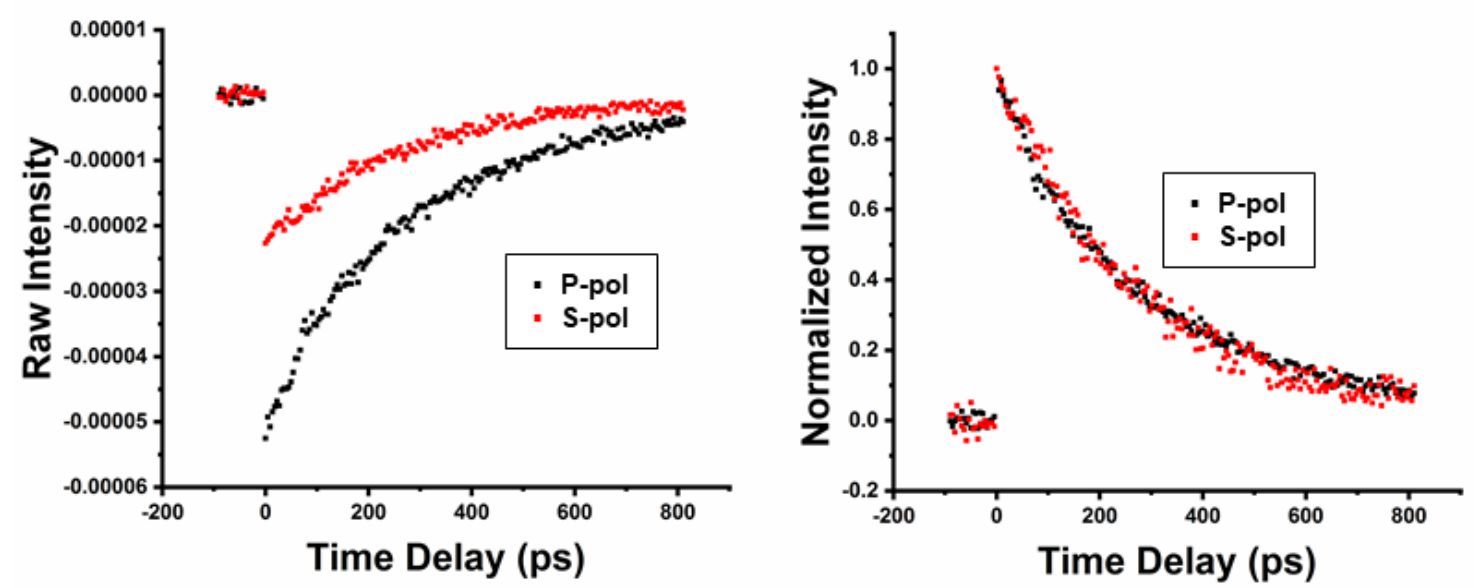

Figure S1: Excited state dynamics of 1 mivı IK-144 in metnanoı comparing airierences in the signal as a function of the incident beam polarizations. Signal in the red traces was generated with both the pump and probe incident on the sample interface with s-polarization while the black traces show signal from $p$-polarization. The raw intensity is plotted on the left showing larger signal for $p$-polarization while the normalized intensities on the right show that the same dynamics are observed for either polarization state. 


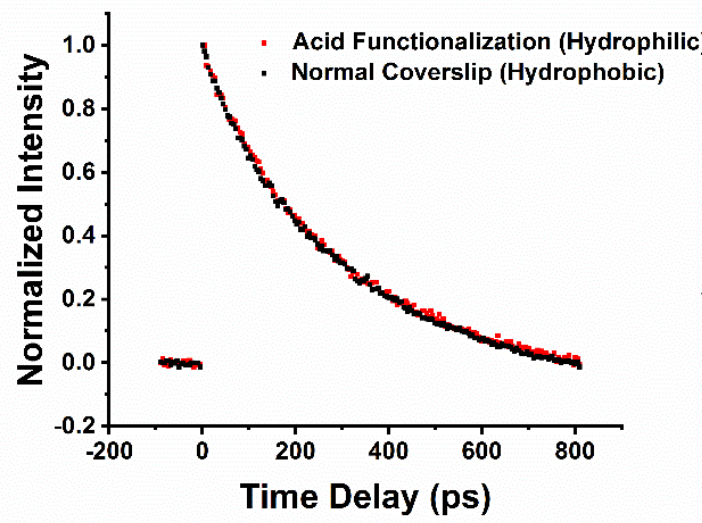

\section{Normal (Hydrophobic)}

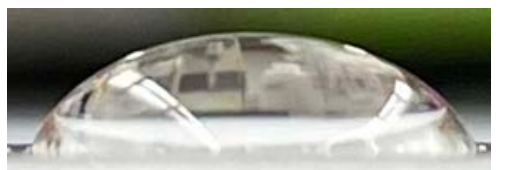

\section{Acid Washed (Hydrophilic)}

Figure S2: Excited state dynamics for $1 \mathrm{mM}$ solutions of IR-144 with normal hydrophobic coverslip and acid washed hydrophilic coverslip. Side profiles of $100 \mu \mathrm{L}$ of water is shown for a depiction of water contact angle.
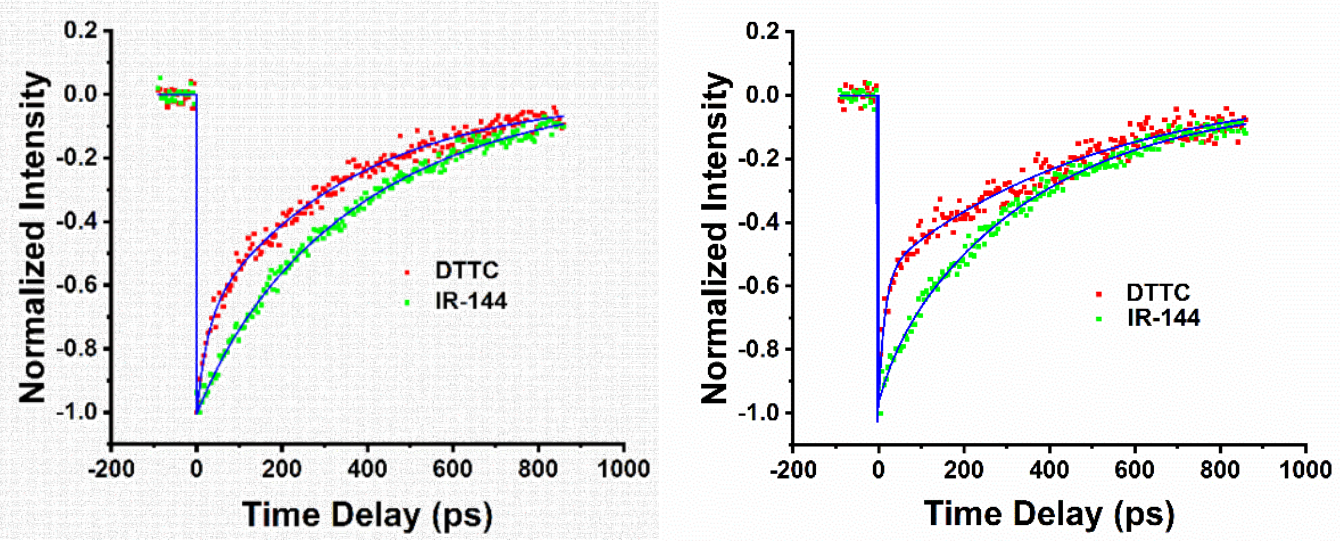

\begin{tabular}{|c|c|c|}
\hline & DTTC & IR-144 \\
\hline A1 & -0.248 & -0.0518 \\
\hline A2 & -0.3 & -0.425 \\
\hline $\mathrm{t} 1$ & 15 & 41 \\
\hline Fit2 & 5.07 & 358 \\
\hline
\end{tabular}

\begin{tabular}{|c|c|c|}
\hline & DTTC & IR-144 \\
\hline A1 & -0.147 & -0.04 \\
\hline A2 & -0.35 & -0.47 \\
\hline t1 & 24 & 77 \\
\hline t2 & 355 & \\
\hline
\end{tabular}

collected in isolated channels in regions I and II (left) or in the mixed channel in region III (right). The extracted fit parameters are shown in the tables below each plot where A1 and $\mathrm{A} 2$ are the amplitudes for the $\mathrm{t} 1$ and $\mathrm{t} 2$ lifetime components (in ps), respectively. 


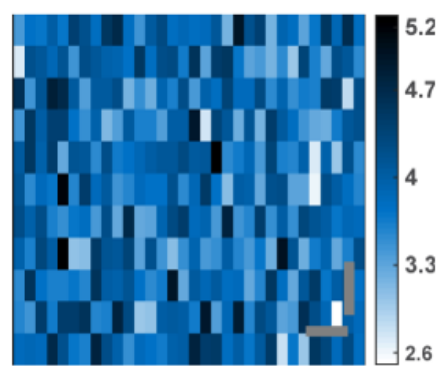

$\Delta \mathrm{t}=-13 \mathrm{ps}$
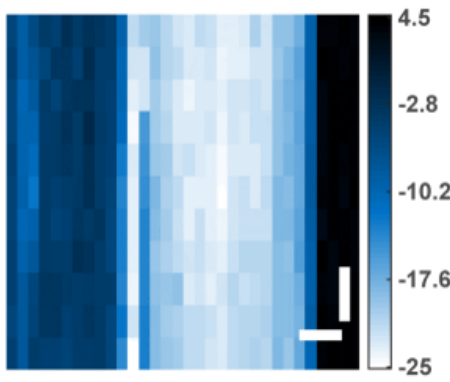

$\Delta \mathrm{t}=+10 \mathrm{ps}$

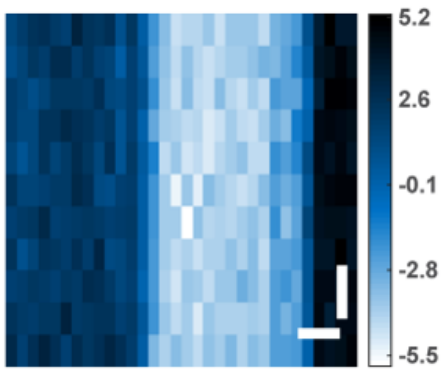

$\Delta \mathrm{t}=+400 \mathrm{ps}$

Figure S4: TIRTAM images of DTTC and IR-144 in methanol at different delay times. On the left, no signal is observable at -13 ps before time zero. In the center, 10 ps after time zero, there are three distinct regions of signal with the medium blue signal of DTTC, white high signal of IR-144, and tape in the black region. These signals are still visible at 400 ps after time zero albeit with lower overall signal as seen on the right.

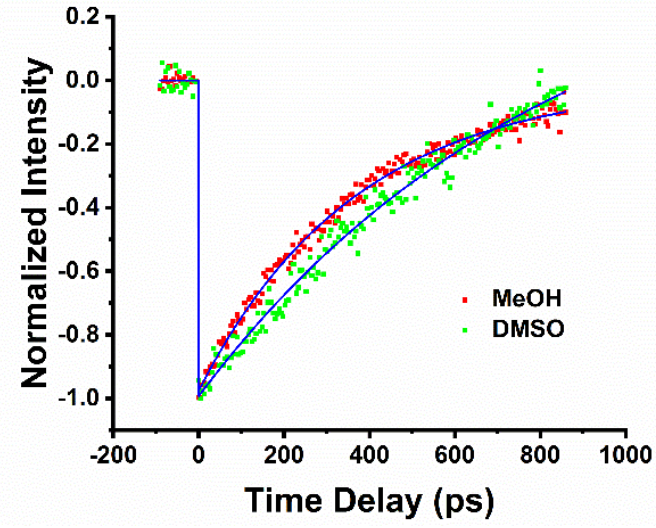

\begin{tabular}{|c|c|c|}
\hline & Methanol & DMSO \\
\hline A1 & -0.487 & -0.742 \\
\hline t1 & 369 & 829 \\
\hline
\end{tabular}

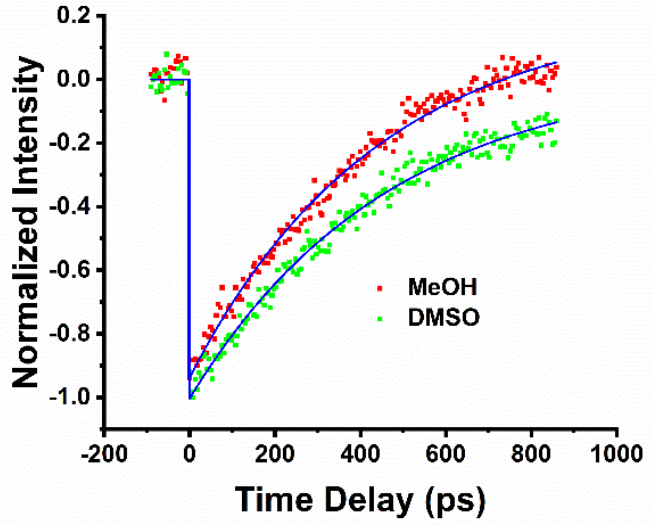

\begin{tabular}{|c|c|c|}
\hline & Methanol & DMSO \\
\hline A1 & -0.582 & -0.515 \\
\hline t1 & 443 & 463 \\
\hline
\end{tabular}

Figure S5: Excited state dynamics for $1 \mathrm{mM}$ solutions of IK-144 in methanol and/or DIVISU collected in isolated channels in regions I and II (left) or in the mixed channel in region III (right). The extracted fit parameters are shown in the tables below each plot where A1 is the amplitude for the $\mathrm{t} 1$ which is the lifetime (in ps). 


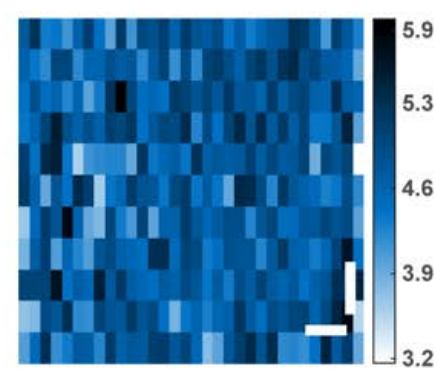

$\Delta \mathrm{t}=-13 \mathrm{ps}$

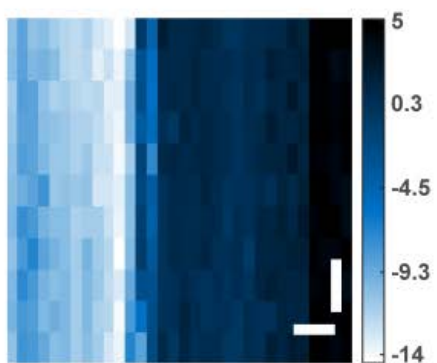

$\Delta \mathrm{t}=+10 \mathrm{ps}$

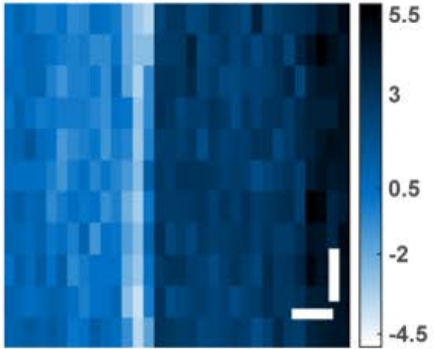

$\Delta \mathrm{t}=+400 \mathrm{ps}$

Figure S6: TIRTAM images of IR-144 in methanol and DMSO at different delay times. On the left, no signal is observable at -13 ps before time zero. In the center, 10 ps after time zero, there are three distinct regions of signal with the medium blue signal of IR-144 in DMSO, white high signal from IR-144 in methanol, and tape in the black region. These signals are still visible at 400 ps after time zero albeit with lower overall signal as seen on the right. 